\title{
A STUDY OF EIGHT SURGICALLY TREATED CASES OF SPONTANEOUS SUB-CORTICAL HAEMATOMA*
}

\author{
BY \\ ALOYS WERNER \\ From the Neurosurgical University Clinic, Ziirich
}

It is almost half a century since Cushing (1903) reported the first surgical removal of an intracerebral blood clot. His report was followed six years later by a similar account by Russell and Sargent (1909). And yet, apart from sporadic but valuable reports (Penfield, 1933), the neurosurgical era of the treatment of intracerebral haemorrhages may be said only to begin with Craig and Adson's paper (1936) on nine cases. Since then there has been an increasing number of publications about the surgical possibilities in such cases and about the puzzling problems of aetiology and pathogenesis. Jewesbury (1947) reviewed the English literature and added one case of spontaneous intracerebral bleeding cured by operation. From the nonEnglish-speaking countries we should like to mention the efforts made by French neurosurgeons to isolate the spontaneous intracerebral haemorrhage as a clinical entity (David and Hécaen, 1945 ; Guillaume and Joinville, 1945) suitable for neurosurgical treatment, also the studies by Italian neurologists to forward operative treatment of intracerebral haemorrhagic lesions (Fazio, 1950). In reporting these eight cases we wish to contribute to the clinical knowledge, recognition, and timely treatment of the non-traumatic, spontaneous sub-cortical haematoma which, we feel, exists as a clinical entity, while proofs of a uniform aetiology and pathogenesis are still wanted.

\section{Report of Cases}

Case 1. + -Charles $F$., aged 33 , a musician, began to complain of severe headache in the middle of June, 1945, maximal behind the right eye; he noticed also some awkwardness of the fingers of the left hand. On June 30,1945 , there was a sudden onset of paralysis of the fingers on the left hand; next day the headache increased suddenly and violently and was located mostly in the right temporo-parietal region. On July 2 the patient

* Read in part at a meeting of the Society of British Neurological Surgeons, Zürich, on June 6, 1952, and before the Société Médicale de Genève on October 7, 1952.

† This case has been cited by Krayenbühl (1950). vomited and fell unconscious. He was admitted to the medical University Clinic in Zürich, and a lumbar puncture revealed bloodstained cerebrospinal fluid under a pressure of $150 \mathrm{~mm}$. of water. The patient was transferred to the Neurosurgical Department the same day.

His personal and family history contributed nothing.

Examination on July 2, 1945, gave a blood pressure reading of $130 / 70 \mathrm{~mm}$. $\mathrm{Hg}$. The patient was very drowsy, and there was marked stiffness of the neck. Fundi were normal and there was complete left homonymous hemianopia, left lower facial palsy, and left-sided spastic hemiplegia, with hyperactive tendon jerks and extensor plantar responses. Sensibility could not be accurately tested.

Arteriogram.-An arteriogram on July 2 showed good filling of the anterior and posterior cerebral arteries on the right ; the middle cerebral artery was filled only for a short distance, where some opaque substance seemed to have escaped from the vessel (Fig. 1).

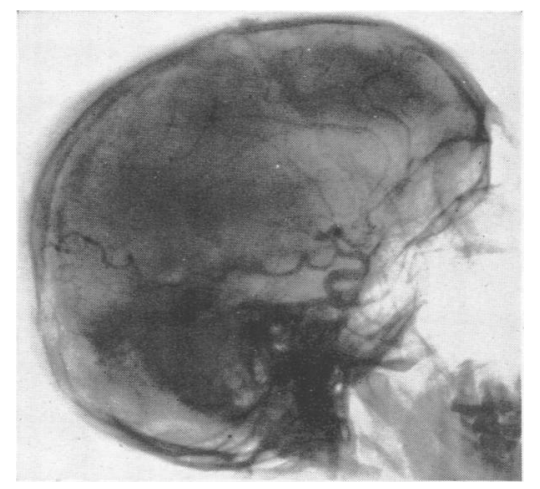

FIG. 1.-Case 1: Right cerebral angiogram showing partial occlusion of the branches of the middle cerebral artery. The anterior and posterior cerebral arteries are well filled; slight stretching of the pericallosal artery.

Pneumoencephalogram.-A first investigation on July 25 disclosed a marked shift to the left of the third and lateral ventricles, which were dilated (Fig. 2a). A repeat air study on August 17 still showed a dilatation of the lateral ventricles; the third ventricle was less shifted. 


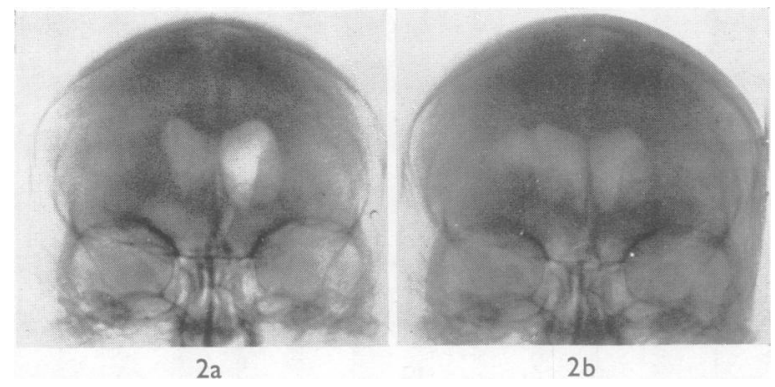

FIG. 2a.-Case 1: Air study three weeks after the illness began, showing marked shift of the ventricular system to the left, especially of the third ventricle.

FIG. 2b.-Case 1 : Air study three weeks later. A huge paraventricular air-filled intracerebral cavity can be seen communicating with the right lateral ventricle.

The whole frontal horn on the right was compressed from the lateral aspect. There was an intracerebral air collection communicating with portion 3 of the right lateral ventricle (Fig. 2b).

Pre-operative Course.-Within the first week the patient regained full consciousness, the hemiplegia regressed, more in the leg than in the arm, but the homonymous hemianopia did not improve. At the end of six weeks he was able to walk alone.

Operation.-Professor H. Krayenbühl performed a right osteoplastic fronto-temporal craniotomy. The dura was under tension which was released by tapping the cyst and aspirating about $20 \mathrm{ml}$. of clear yellow fluid. After opening of the dura the frontal and temporal gyri were not seen to be particularly flat or wide. After excision of a small circular zone of cortex and white matter in the postero-lateral frontal region at a depth of about $3 \mathrm{~cm}$. a cavity was entered measuring $9 \times 5 \times 4 \mathrm{~cm}$.; its walls were about $2 \mathrm{~mm}$. thick and shiny. The cyst contained greyish-red fibrincoagula and about $50 \mathrm{ml}$. of fluid. In the bottom of the cavity there was a spherical mass covered with fibrin and containing numerous fine arterial vessels. This mass was excised. The dura was completely closed.

Histological Examination.-The excised mass comprised fibroblastic tissue containing much haemosiderin and haematoidin, small and large vessels, but no neoplastic tissue.

Post-operative Course.-This was uneventful except for an immediate post-operative increase of the hemiplegia. At the time of discharge on September 17 the patient could walk with a stick, with a spastic-ataxic gait, but he could not use the left arm or hand.

The clinical picture on admission was that of a subarachnoid haemorrhage. As the likeliest diagnosis was a ruptured aneurysm, it was thought, as pointed out elsewhere by Krayenbühl (1950), that the aneurysm had probably thrombosed. However, the course in hospital during the first weeks corrected this impression, signs of a spaceoccupying lesion showing up more clearly after the spontaneous recovery from the initial stroke. It is interesting to note that the first pneumoencephalogram three weeks after admission showed only a shift of the ventricular system while the second air study three weeks later disclosed the intracerebral cavity. The extensive nature of the neurological disorders (homonymous hemianopia, sensori-motor hemiparesis) can be accounted for only by the fact that the huge cavity in the posterior frontal region was exerting considerable pressure on neighbouring structures such as the internal capsule.

Case 2.-During the night of Oztober 2, 1945, Josef G., aged 48, a shoemaker, suddenly vomited, and developed a headache and drowsiness. The next day he went to work as usual, but did not know how to use his tools. On October 4 he became subcomatose and was sent to the Psychiatric University Clinic, Zürich, where on admission he gave the impression of being completely drunk. The next two days he was deeply comatose, and the cerebrospinal fluid taken on October 5 was bloodstained. The patient regained consciousness spontaneously on October 7 and felt well after another 24 hours. He was sent for further investigations to the Neurosurgical Clinic on October 18.

His personal and family history contributed nothing.

On examination (October 18, 1945) there were no subjective complaints, and the patient said he did not understand the reason for his transfer to the Neurosurgical Department. The blood pressure was $130 / 90$ $\mathrm{mm}$. Hg. There was slight confusion and some difficulty in finding the right word, slight apraxia, and anosmia on the left. There was no stiffness of the neck, and the fundi, vision, and visual fields were normal. The pupils were equal and reacting normally, eye movements full, and the corneal reflexes brisk. There was slight paresis of the lower facial muscles on the right. There were no

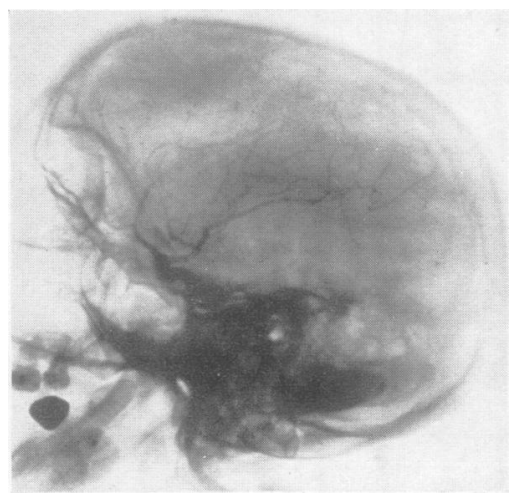

FIG. 3.-Case 2: Left cerebral angiogram. Backward displacement of the proximal course of the Sylvian vessels. 
abnormal findings in the extremities and no impairment of gait. The C.S.F. was under pressure of $305 \mathrm{~mm}$. of water, containing $22 \mathrm{mg}$. of protein per $100 \mathrm{ml}$.; Pandy + ; 2 cells per c.mm.

A pneumoencephalogram on October 19 demonstrated a shift of the ventricles to the right, with amputation of the frontal horn on the left.

An arteriogram on October 26 showed that the left middle cerebral artery was displaced backwards and downwards (Fig. 3).

Operation.-On October 31, 1945, Professor H. Krayenbühl performed a left transfrontal osteoplastic craniotomy, and reported that the dura was not particularly tense but was bluish over the frontal pole. On opening the dura a dark yellow membrane 3-4 mm. thick around the frontal pole was seen; it could be peeled off easily except at the tip of the frontal pole, where it was adherent to the very soft and yellow discoloured cortex. A brain needle was passed through the cortex and entered a cavity at a depth of about $1 \mathrm{~cm}$., from which a few millilitres of dark brown fluid were aspirated. The frontal pole was excised totally after opening a sub-cortical cavity the size of a small orange filled with organized blood clots. The source of the haemorrhage could not be found. The cavity was completely closed.

Histological examination disclosed red softening of the cortex and non-specific thrombosis of the pial arteries.

The post-operative course was uneventful, and the patient left the hospital on November 11, 1945, with neither neurological nor psychiatric disability.

The sudden onset of headache, vomiting, and apraxia, leading to coma after 36 hours, followed by spontaneous recovery, is the leading feature of this case. A pre-operative diagnosis of bleeding into a left frontal tumour had been made. The operative findings seem to indicate that the intracerebral haemorrhage leaked through the tip of the frontal pole into the subdural space. It is of interest that four weeks after the bleeding there were still partially organized blood clots in the cavity.

Case 3.-Robert St.-, aged 26, a farmer, was working in the fields on October 11, 1948, when he suddenly felt a weakness of the left leg, then of the left arm, which became flaccid ; there was no loss of sensibility, no impairment of consciousness, and no pain. He was admitted the following day to a local hospital ; from the fifth day the hemiparesis recovered in the opposite way from which it had developed, but the foot remained paralysed. The patient was then sent for further investigation to the Neurosurgical University Clinic, Zürich, on November 9, 1948.

His personal and family history contributed nothing.

On examination on November 9 the blood pressure was $130 / 90 \mathrm{~mm}$. Hg. There was no psychical disorder. There was slight weakness of the lower face, the arm and the leg on the left, and the foot was still paralysed; there was no impairment of sensation. The cerebrospinal

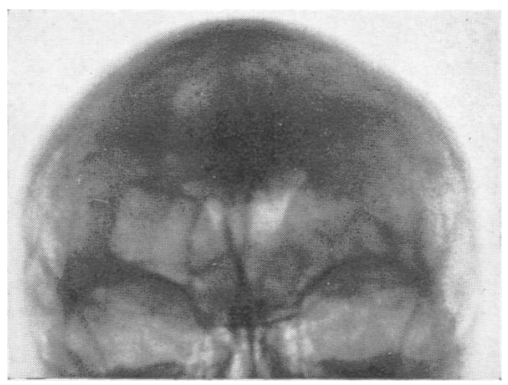

FIG. 4.-Case 3: Air study (A.P. view). There is a large intracerebral air collection communicating with portion 3 of the right lateral ventricle. It is noteworthy that such a huge space-occupying lesion causes no displacement of the ventricular system.

fluid was yellowish but the pressure was not raised.

A pneumoencephalogram on November 10 showed that the left lateral ventricle was slightly dilated; there was no shift of the ventricular system. There was an air-filled intracerebral cavity in the right precentral region (Fig. 4).

An electroencephalogram on November 10 showed some slow waves in the right post-central region (Fig. 5).

Operation.-Professor $\mathrm{H}$. Krayenbühl performed a right fronto-parietal osteoplastic craniotomy on November 19. He reported that the gyri were flattened and soft in the upper part of the precentral region near the midline. A huge, conical cavity was entered one centimetre below the surface, the apex of which opened through a small hole into the lateral ventricle. The walls consisted of a shiny, brownish membrane; neither abnormal vessels nor tumour tissue could be seen. The cyst contained

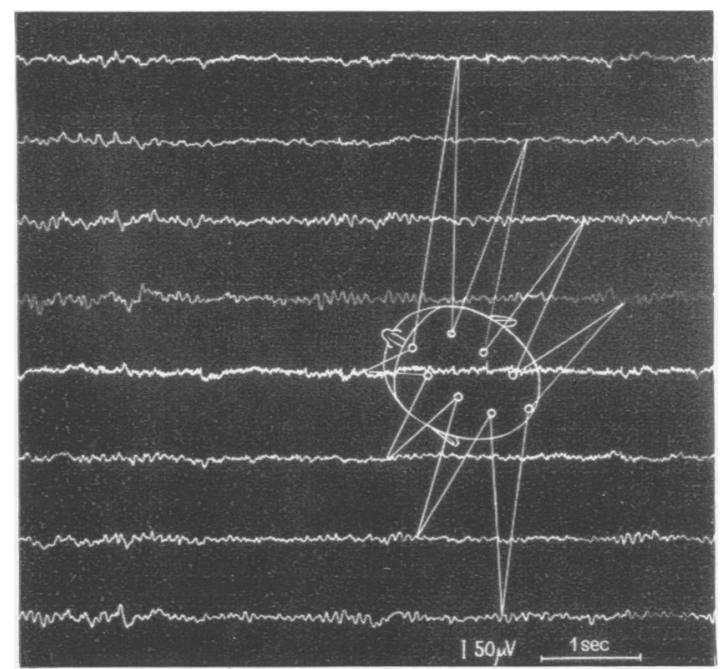

FiG. 5.-Case 3 : E.E.G. tracing taken a month after the onset of the illness. There are some slow waves in the right post-central region. 
about $40 \mathrm{ml}$. of yellow fluid. The membrane was partially removed and the dura closed incompletely.

The post-operative course was uneventful, and the patient was discharged on November 25, 1948, with a partial recovery of the left foot. He was seen again in March, 1952, when he had just had a generalized seizure beginning with head turning to the left but with extensionspasm of the right leg and no loss of consciousness. On examination he showed no neurological disability. His blood pressure was $150 / 100 \mathrm{~mm}$. of water. The E.E.G. showed slow waves in the right central region which were partly due to the bone flap.

The onset of a rapidly increasing hemiplegia without headache, impairment of consciousness, or vomiting, regressing after a few days, could easily have been mistaken for a cerebral thrombosis. The diagnosis was established only by pneumoencephalography. The E.E.G. localized the lesion too far posteriorly.

Case 4.-August P., aged 41, an orderly, had been complaining of slight but constant headache for the past two weeks. On February 7, 1950, he began working as usual at 5.30 a.m., complaining of very violent, rightsided headache. At 8 a.m. he gave up his work because he was letting everything fall ; soon afterwards he became drowsy, developed left-sided hemiplegia, and was brought to the medical wards. Two lumbar punctures on February 8 showed bloodstained C.S.F. The patient was then transferred to the Neurosurgical Clinic as an emergency case.

Personal and Family History.-His father died at 74 of apoplexy, and his mother at 50 of kidney disease. Two brothers complain of headache. The patient himself had suffered from headaches since childhood. He had gastroenteritis in 1942, and eosinophilic pleurisy in 1946 since when he had had rheumatic pain in the fingers. He had left trigeminal neuralgia in 1948.

Examination.-On February 8 his blood pressure was $80 / 45 \mathrm{~mm}$. Hg. He was drowsy. There was no stiffness of the neck, and the fundi were normal. There was no impairment of visual fields, the pupils reacted and were equal. There was left hemiparesis, more marked in the upper limb. The drowsiness deepened on February 9 and 10.

An electroencephalogram on February 9 revealed severe bilateral abnormality, specially marked in the right anterior temporal region.

An arteriogram on February 9 showed the anterior cerebral artery to be stretched and shifted to the left (Fig. 6).

Operation.-Dr. G. Weber performed a right frontotemporal osteoplastic craniotomy on February 11. He reported that the dura was under tension which was released by tapping the right lateral ventricle. The gyri were widened and there was a sub-cortical cavity containing blood coagula and measuring $7 \times 6.5 \times 3 \mathrm{~cm}$. occupying the whole frontal lobe. No connexions between cavity and surface or cavity and ventricle could

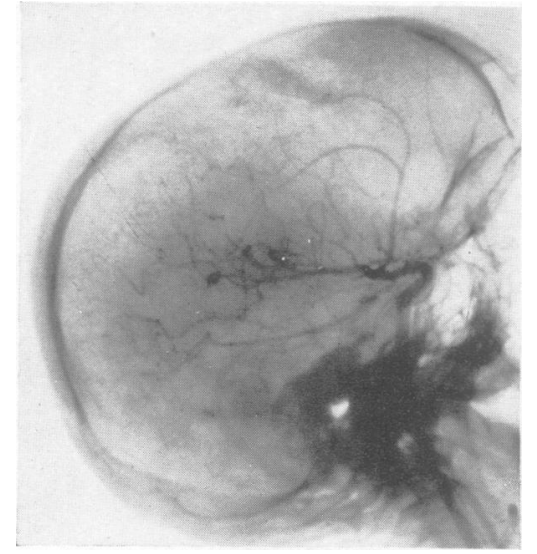

FIG. 6.-Case 4: Right-sided cerebral arteriogram. Stretching of the anterior cerebral artery; moderate backward shift of proximal portions of the Sylvian vessels.

be found. The walls were made of white matter and no abnormal vessels or tumour tissue were seen. The dura was completely closed.

Post-operative Course.-The patient recovered from his comatose state while still on the operating table. On the following day he was still confused but responded well. On February 14 he had an epileptic fit beginning in the left corner of the mouth. On the following day his temperature rose and he became comatose and died the next day with signs of cardiovascular and respiratory failure and with high fever.

Necropsy.-There was a pressure cone into the foramen magnum. There was no haemorrhage or vascular abnormality. The cause of the right frontal sub-cortical bleeding could not be ascertained.

This patient had had constant slight headache for two weeks which quite suddenly became excruciating and unilateral, leading to drowsiness and coma, with hemiplegia within three hours. Bloodstained C.S.F. proved the subarachnoid haemorrhage. A ruptured aneurysm was thus the probable cause, and an arteriogram was done which disclosed the right frontal expanding lesion. The necropsy did not explain why the patient, having recovered from the comatose state (for the second time) after the operation, developed a pressure cone into the foramen magnum three days later and died. Nor could the pathologist find the reason for the sudden intracerebral bleeding.

Case 5.-Erika K., aged 43, a housewife, was normally delivered of a second child after a pregnancy during which she suffered much from headache and vomiting. On the sixth day there was a sudden onset of very severe right-sided headache and vomiting and, a few hours 
later, of a left-sided complete hemiplegia, without loss of consciousness. There was bloodstained cerebrospinal fluid, and with the diagnosis of a ruptured aneurysm the patient was sent to the University Clinic at Zürich on February 27, 1951.

Personal and Family History.-The father'died aged 55 from apoplexy, and the mother has high blood pressure. The patient had articular rheumatism in 1919, but had remained well since then, but for occasional headaches. Her first pregnancy was in 1948.

Examination.-On February 27 the blood pressure was $135 / 80 \mathrm{~mm}$. $\mathrm{Hg}$. She was drowsy, with "déviation conjugée" of the eyes and head to the right, a leftsided homonymous hemianopia, left lower facial palsy, and flaccid paralysis of the left arm and leg, with abolition of all kinds of sensation.

Arteriogram.-An arteriogram on February 28 showed stretching of the arteries around the Island of Reil, and local narrowing of the anterior cerebral artery just distal to its bifurcation from the carotid artery (Fig. 7).

Pneumoencephalogram.-A pneumoencephalogram on March 1 showed a sub-cortical multilocular cavity in the right central region, and a shift of the ventricular system to the left (Fig. 8).

Operation.-On March 5 Professor H. Krayenbühl performed a right fronto-parietal osteoplastic craniotomy. He reported that the dura was tense. The cortex showed a localized yellowish discoloration in the lateral precentral region. At a depth of less than one centimetre the brain needle entered a huge cavity from which $20 \mathrm{ml}$. of a brownish fluid was withdrawn. This relieved the intracerebral pressure. An incision $2 \mathrm{~cm}$. long was made in the foot of $F_{3}$, coagulated blood being evacuated. The cavity extended down to the ventricle wall. Gelfoam was used for haemostasis of small bleeding points along the cavity wall. The dura was completely closed.

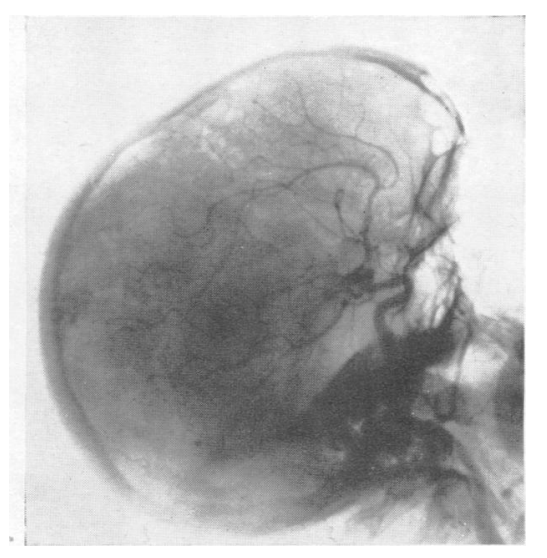

Fig. 7.-Case 5: Right-sided cerebral arteriogram. Separation of the fronto-parietal ascending branches of the middle cerebral artery.

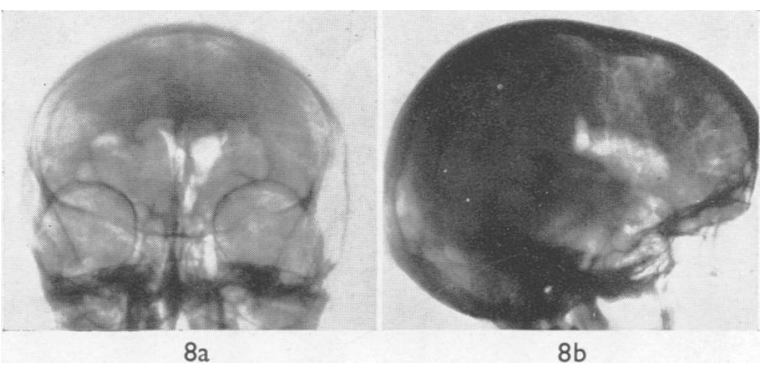

Fig. 8a.-Case 5 : A.P. view of air study.

FIG. 8b.-Case 5 : A.P. lateral view of air study. Slight shift to the left of the ventricular system. Two intracerebral air-filled cavities are seen in the lower part of the right precentral region.

Post-operative Course.-The hemiplegia slowly recovered. After one year the patient is able to walk with a stick for a mile or so. She still complains of headache. Her blood pressure remains normal.

Occurring six days after childbirth, this sudden hemiplegia might have been considered due to a venous thrombosis but for the findings of a subarachnoid haemorrhage. On the other hand, the clinical picture with "déviation conjugée", complete hemiplegia, and homonymous hemianopia, looked very like a capsular hemiplegia. As the patient was still relatively young, a diagnosis was made of a ruptured aneurysm. As in Case 1, the extent of the neurological disorders is here clearly explained by the localization of the intracerebral haematoma within the central region reaching down to the lateral ventricle. This case provides good reason to think that capsular haemorrhages too may be treated surgically with success, as reported by Guillaume and others, and advocated by Fazio.

Case 6.--Hélène S., aged 52, a secretary, had her first epileptic fit while at work on December 3, 1949: she became stiff, the right arm was shaky, the head extended backwards, the face became cyanotic, and the respirations stertorous. The patient was sent to a psychiatric clinic where a diagnosis of a neurotic fit was made, the neurological examination being normal. The blood pressure then was $200 / 100 \mathrm{~mm}$. Hg. From then on the patient complained at times of attacks of headache with dizziness. She went on working without interruption until she had a second generalized seizure on April 25, 1951, while having lunch in a restaurant; this was accompanied by loss of consciousness, biting of the tongue, and incontinence. She was sent to the Medical Clinic in Zürich on the same day and transferred to the Neurosurgical Clinic five days later.

Personal and Family History.-Her mother died aged 32 from heart disease. The patient had already suffered from headache for some years previously.

Examination.-On April 30 her blood pressure was $135 / 90 \mathrm{~mm}$. Hg. Consciousness was slightly impaired, 


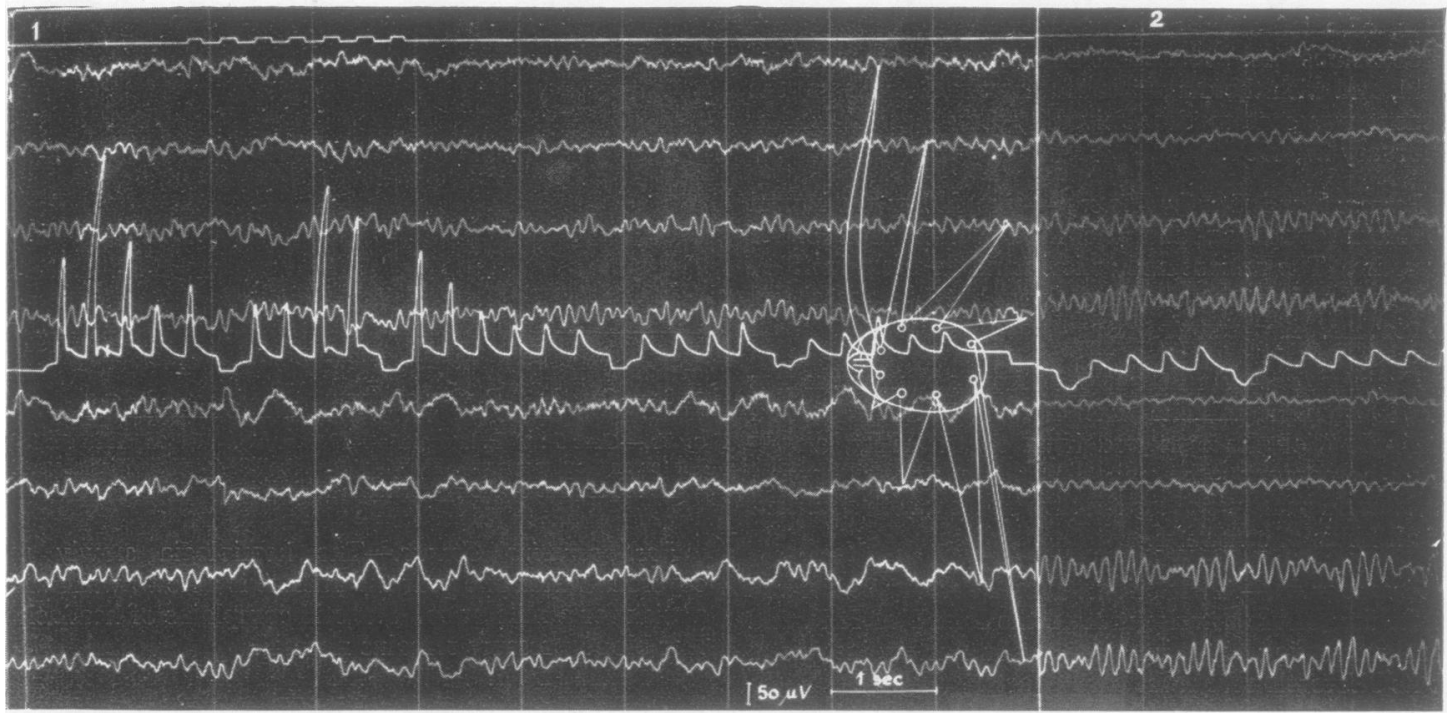

FIG. 9.-Case 6: 1. Pre-operative E.E.G. shows a severe abnormality on the left side, specially marked in the lower part of the left precentral region. 2. Post-operative E.E.G. shows pronounced improvement of the electrical activity on the affected side.

with disorientation in time, difficulty in reading, writing, and calculating. The neck was slightly stiff but Lasègue and Kernig's sign was negative. There was no anosmia, the fundi were normal. There was a right homonymous hemianopia, but pupils and eye movements were normal. She had slight facial weakness on the right of a central type but no other abnormal neurological signs.

Electroencephalogram.-Electroencephalograms on April 30 and May 5 showed severe abnormality on the left with a maximum in the anterior temporal region (Fig. 9, 1).

Arteriogram.-An arteriogram on May 1 showed stretching of the left anterior cerebral artery, and forward displacement of the Sylvian group and terminal branches of the posterior cerebral artery (Fig. 10a).

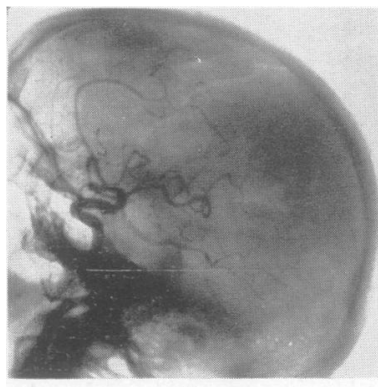

$10 \mathrm{a}$

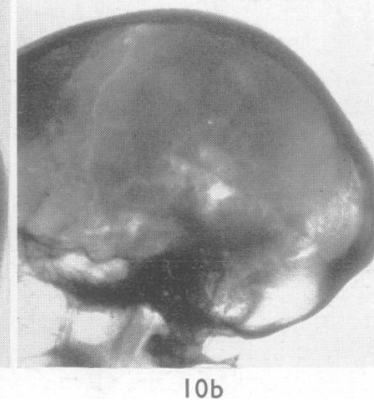

FIG. 10a.-Case 6: Left cerebral angiogram showing a stretching of the anterior cerebral artery, a shift forward of the Sylvian group which has the "spring" appearance commonly seen in occipital space-occupying lesions.

Fig. 10b.-Case 6 : Air study, P.A. lateral view. Portion 4 and occipital horn of the left lateral ventricle are pushed downward.
Pneumoencephalogram.-A pneumoencephalogram on May 2 showed a shift of the left occipital horn downwards and to the right (Fig. 10b).

Operation.-On May 9 Professor H. Krayenbühl performed a left parieto-occipital osteoplastic craniotomy. He reported that the dura was moderately tense. The posterior parietal gyri showed a brownish discoloration, and the cortex there was extremely thin. More laterally the gyri were widened and flattened, partly greyish-red. Towards the occipital lobe there was a vascular mass the size of a cherry draining into dilated veins. Sub-cortically the mass communicated with a huge cavity, $5 \times$ $6 \times 6 \mathrm{~cm}$., filled with clotted blood, separated from portion 4 of the lateral ventricles only by a thin membrane. The dura was completely closed.

Histological Report.-There was sub-cortical bleeding by markedly arteriosclerotic cerebral and meningeal vessels, but no neoplastic growth.

Post-operative Course.-The patient made an uneventful recovery, and left the hospital on May 25 still showing a complete right-sided homonymous hemianopia and difficulty in calculating and writing. A control E.E.G. on August 28 showed a marked improvement (Fig. 9). On February 25, 1952, the patient was seen again. She had been unable to resume her work as a secretary because she still showed mental deterioration : reading, writing, calculating were greatly impaired. Apart from the right homonymous hemianopia, no abnormal neurological signs were found.

This case shows that arteriosclerotic patients may have other than capsular haemorrhages. The cerebral arteriosclerosis may account for the peculiar onset with a generalized seizure, the 
diseased brain reacting to a sudden change in intracranial pressure with an epileptic discharge, as it had already done one year previously.

Case 7.-Max H., aged 36, a workman, suddenly vomited and fell unconscious on October 2, 1951, then showed spasms of the extremities. In the evening he responded again. He was sent to a local hospital on October 4. On admission there he showed a complete disorientation, stiffness of the neck, hyperactive tendon jerks, and bilateral extension of the big toes. His blood pressure was $105 / 60 \mathrm{~mm}$. Hg. A lumbar puncture disclosed bloodstained C.S.F. under a pressure of 230 $\mathrm{mm}$. of water. On the following day the patient was transferred to the Neurosurgical Clinic at Zürich. During the previous weeks he had been complaining of some headache and dizziness.

At the age of 11 he had had an accident to his leg, and hepatitis at an unknown age.

On examination on October 5 he was markedly disorientated in space and time, and about himself. The neck was stiff and he had a homonymous hemianopia on the right ; the left pupil showed no reaction to light and the right was very sluggish. He had slight spastic paresis of the right leg.

Arteriogram.-An arteriogram on October 5 showed that the left middle cerebral artery was shifted upwards and forwards (Fig. 11a).

Electroencephalogram.-A first record showed a marked asymmetry between both hemispheres, the left side being abnormal with a maximum in the frontal region. A week later the whole record showed an increased bilateral abnormality, now more pronounced on the left side, from the frontal to the temporal region.

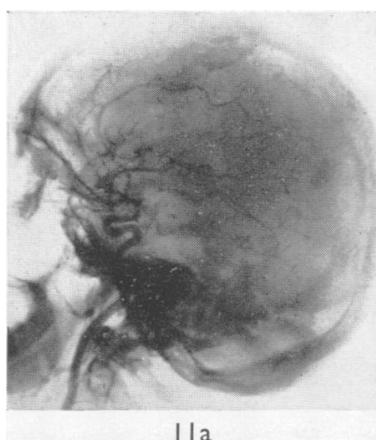

II a

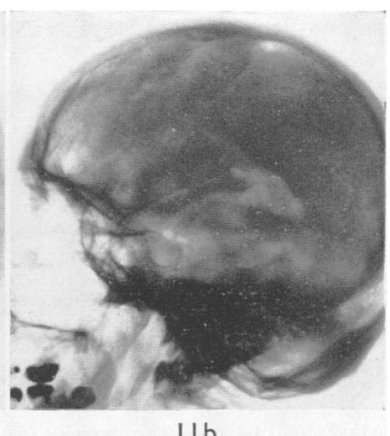

IIb

FIG. 11a.-Case 7 : Left cerebral angiogram. Upward displacement of temporal branches of the Sylvian vessels.

FIG. 11b.-Case 7 : Air study, lateral view. Trigone and posterior portion of temporal horn are curved upwards, being displaced from below.

A third recording another week later showed a practically normal activity on the right hemisphere ; on the left, especially in the temporal region, there was still a marked abnormality, that is, a pronounced depression of the electrical activity (Fig. 12).

Pneumoencephalogram.-A pneumoencephalogram on October 16 showed a shift of the entire ventricular system to the right, with marked elevation of the left temporal horn. There was a suspicion of a mid-line space-occupying lesion (Figs. 11b and 13a). The C.S.F., taken on this occasion by cisternal puncture, was under normal pressure, showed $149.5 \mathrm{mg}$. of protein per 100 $\mathrm{ml}$., and contained about 100 white cells per c.mm.

Operation.-On October 29 Professor H. Krayenbühl performed a left temporo-occipital osteoplastic cranio-

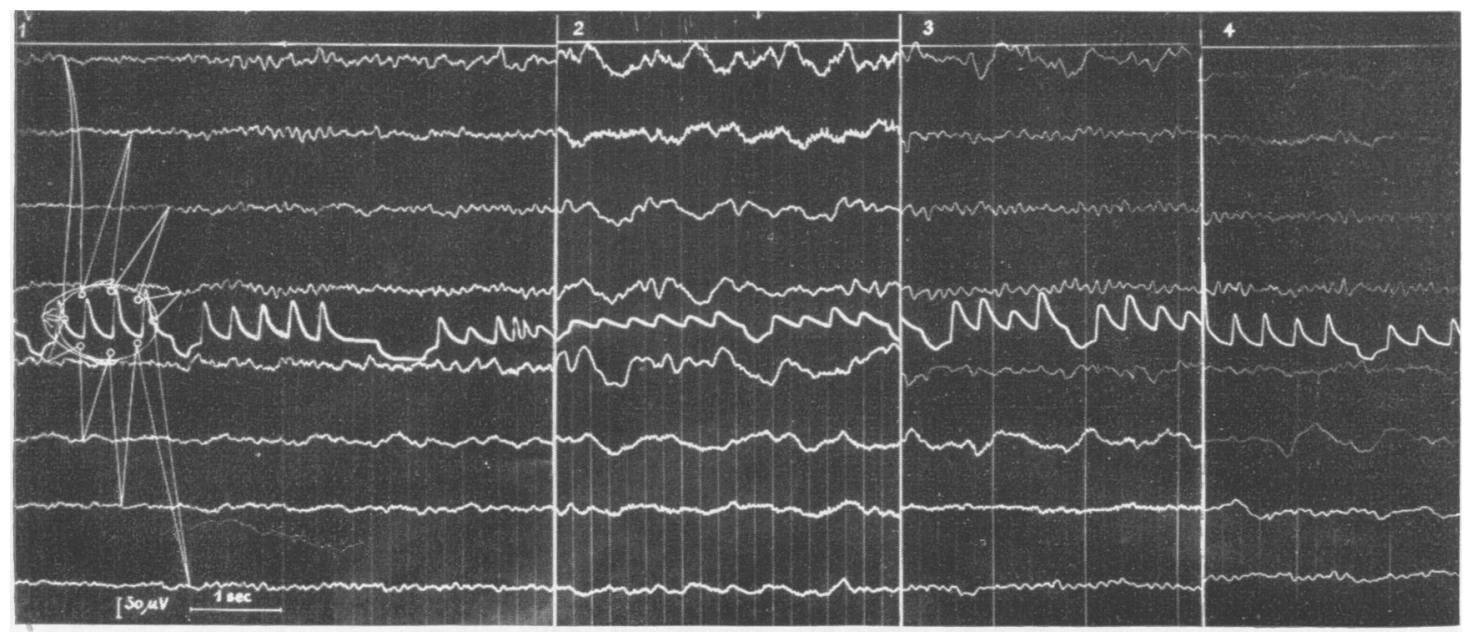

Fig. 12.-Case 7 : 1, 2, and 3 are pre-operative E.E.G. tracings. In 1, marked asymmetry between both hemispheres; in 2, a week later, the abnormality has spread to both hemispheres; in 3 , another week later, the right hemisphere now again shows a practically normal activity; in 4 , a post-operative tracing still shows slow waves on the affected side. 

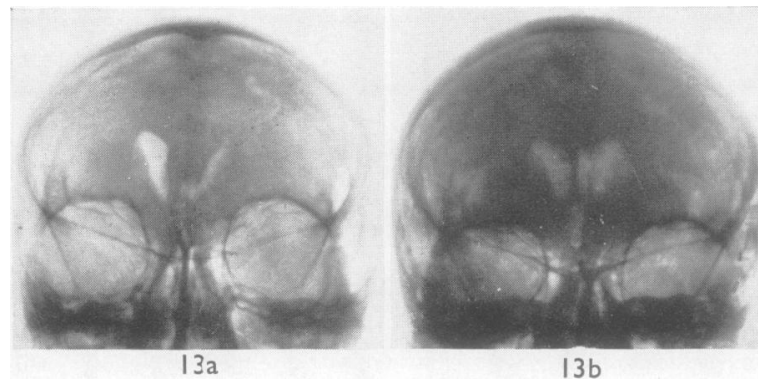

Fig. 13a.-Case 7 : Pre-operative A.P. encephalogram, showing shift to the right of the right lateral ventricle. The air shadow on the left was thought to be the left lateral ventricle displaced by a medial space-occupying lesion.

Frg. 13b.-Case 7 : Post-operative A.P. encephalogram showing normal situation of right lateral and third ventricles; the left lateral ventricle is slightly dilated but normally located.

tomy. He reported that the dura was not very tense. The first and second temporal gyri were flattened and abnormally wide, and showed a brownish-yellow discoloration. There was a sub-cortical cavity the size of an orange containing coagulated blood. The cavity reached the ventricular wall, which was not ruptured, and extended into the occipital lobe. After the haematoma had been taken out there remained, dorsal to the temporal horn, a greyish-red mass which was partially excised for microscopic examination. The dura was completely closed.

Histological Report.-The exised discoloured tissue proved to be granulation tissue with organized haemorrhage.

Post-operative Course.-This was uneventful. The patient was discharged on the sixteenth post-operative day, still showing a marked psycho-organic syndrome, an impairment of the right visual field, and a slight hemiparesis on the right side. A pneumoencephalogram performed on the fourteenth post-operative day showed no shift of the ventricular system, but the left lateral ventricle was wider than the right (Fig. 13b). Four months later the patient still complained of impairment of memory. On examination the right visual field was not yet complete, but the right-sided hemiparesis had completely recovered.

This patient, admitted with the presumptive diagnosis of a ruptured aneurysm, showed signs of a left temporo-occipital lesion. However, the A.P. view in the air study was very confusing, leading one to suspect also some pathological process in the septum pellucidum. The persistence of a marked psycho-organic syndrome after the emptying of the intracerebral haematoma in the temporooccipital region reinforced the possibility of such a second lesion, which was, however, excluded by the repeat air study two weeks after the craniotomy.

Case 8.*-Georges B., aged 50, a pharmacist, had been helping his gardener to cut down trees on November 9,

\footnotetext{
* This case has been cited by Werner, Babel, and Richard (1952).
}

1951 ; he had had to pull very hard on a rope which he held on his shoulder, and felt very tired from the physical strain. Three hours later while the patient was in his pharmacy, there was a sudden onset of violent headache in the right parieto-occipital region without nausea or impairment of consciousness. He noticed at once that he could not find his way to the left. Shortly afterwards a physician noted stiffness of the neck and a blood pressure of $220 \mathrm{~mm}$. $\mathrm{Hg}$ systolic. The headache and homonymous hemianopia persisted. A neurologist was called who sent the patient to the Clinique Beaulieu, Geneva, on November 14.

Personal and Family History : Three years previously the patient had fallen on the back of his head, but had not hurt himself. For years he had noticed a pulsating vessel in the occipital region, at times slightly painful on pressure. During the last six months he had complained of occasional right-sided headache.

Examination.-Examination on November 14 gave a blood pressure reading of $145 / 90 \mathrm{~mm}$. $\mathrm{Hg}$. There was definite stiffness of the neck, very slight blurring of the optic discs, complete homonymous hemianopia to the left (Fig. 15), and slight left hemiparesis. A lumbar puncture showed a dark yellow fluid under a pressure of $360 \mathrm{~mm}$. of water.

Arteriogram-An arteriogram on November 15 showed that the middle and posterior cerebral arteries were pushed forwards. There was no visible aneurysm, but an extremely large occipital artery (Fig. 14a).

Pneumoencephalogram.-On November 15 sub-occipital puncture in the sitting position was made : the A.P. view showed a dilatation of the left ventricle and a slight shift to the left of the third and lateral ventricles. In the P.A. view there was a marked shift forward of the right occipital horn, which seemed to communicate with a multilocular sub-cortical collection of air (Fig. 14b).

Operation.-On November 22 I performed a right parieto-occipital osteoplastic craniotomy after partial

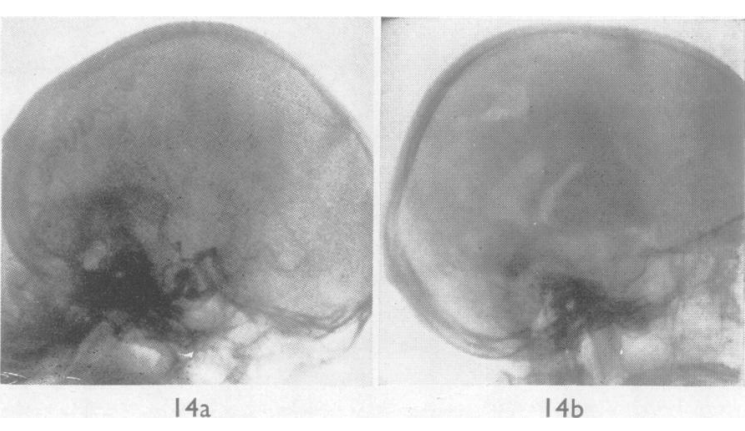

FiG. 14a.-Case 8: Right-sided cerebral angiogram. Both intra- and extra-cranial vessels have filled. There is a forward displacement of the cerebral vessels, most marked of the Sylvian group, with " spring" appearance. Abnormally wide occipital artery.

Fig. 14b.-Case 8 : Air study, A.P. lateral view. Large subcortical air collection pushing forward the right lateral ventricle. 
excision of a greatly dilated occipital artery. Tapping of the left ventricle through a separate burr-hole relieved the intracerebral pressure. The dura appeared normal ; the exposed gyri were flattened and widened and showed a yellowish-red discoloration. The brain had a normal consistency towards the central region, but it was markedly softened near the occipital lobe and the midline. A brain needle was passed towards the ventricle and at one centimetre below the surface entered a cavity from which chocolate-like fluid was easily aspirated. A conical excision of cortex and white matter was then non-neoplastic proliferation of cortical astrocytes and hyalinosis of meningeal arteries. The occipital artery showed a marked fibrous proliferation of the intima, the lumen being very irregular. The elastica was ruptured in many places. There was no inflammatory reaction.

Post-operative Course.-This was uneventful. There was slight aggravation of the left-sided hemiparesis for four days, then a steady improvement. The patient was discharged on December 12 able to walk normally. The hemianopia had already improved in the upper

FIG. 15.-Case 8: Pre- and post-operative visual fields. (The post-operative fields were tested by Dr. Babel on the Maggiore perimeter.) The pre-operative fields were tested as accurately as possible by the author by the confrontation method.
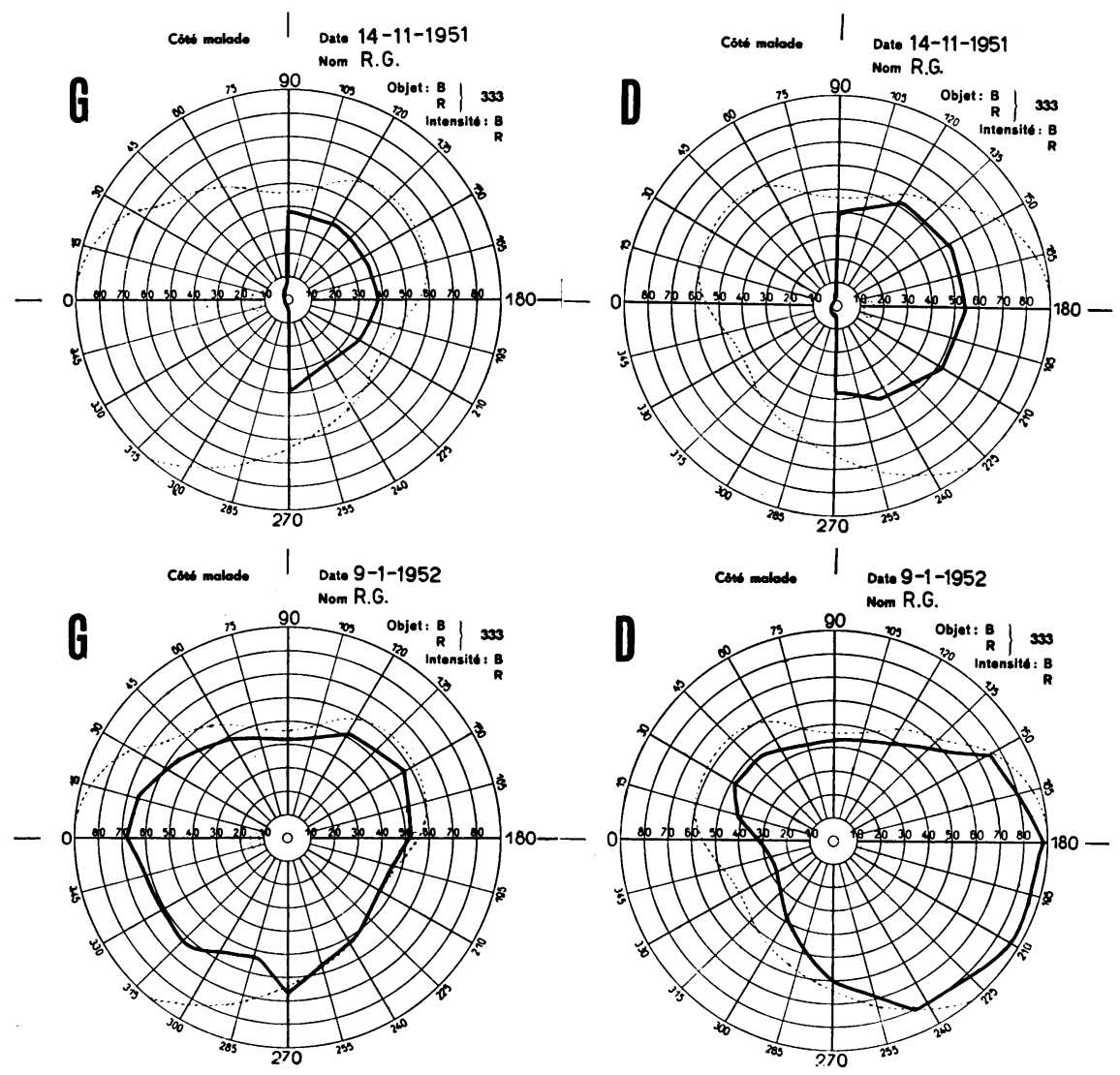

made and the cavity emptied of numerous blood clots. The cavity extended into the occipital lobe for a distance of $6 \mathrm{~cm}$., was about $4.5 \mathrm{~cm}$. wide and $3 \mathrm{~cm}$. high, and reached forward to the ventricular wall. There was no membrane, and the walls were made of yellow, discoloured white matter. Neither neoplastic tissue nor vascular anomaly were to be seen. The dura was completely closed.

Histological Report.-The excised piece of brain showed many perivascular haemorrhages; there was a

quadrant, and by February, 1952, the visual fields were normal (Fig. 15). By the beginning of March the patient had resumed his professional duties.

Various blood analyses were made in order to ascertain whether a blood disease could be incriminated. Blood pressure remained normal throughout. Blood counts were normal, as were tests of coagulability. Thus there were no signs of a haemorrhagic diathesis.

The complete recovery of the hemianopia shows that an intracerebral bleeding, even if very large, 
does not necessarily destroy all the white matter but separates the nerve fibres. ${ }^{*}$

\section{Clinical Analysis}

Incidence.-We are unable to estimate the incidence of spontaneous sub-cortical haematoma mainly because the selected patients seen by the neurosurgeon give no idea of their real frequency.

Age and Sex.-This small group is made up of six men and two women. The youngest patient was a man of 26 ; the oldest a woman of 52 . The average age for men is 39 , for women 47 . Thus it seems that spontaneous sub-cortical haematoma occurs in relatively young patients, as emphasized by Jewesbury (1947), among others, and three times more frequently in men than in women. This agrees with reports by various authors: two-thirds of Craig and Adson's (1936) nine patients were under 40 ; the average age of Klemme's (1941) six patients was 34. Six out of eight patients described by Pilcher (1941) were middle-aged, three being under 20 (but four cases were post-traumatic). Also, Guillaume and Joinville's (1945) patients were mainly between 40 and 45 . But, as reported by Jewesbury (1947), David and Hécaen (1945), de Paula Nogeira (1949), and others, spontaneous intracerebral bleeding can also occur in older people showing no signs of cardiovascular disease.

Mode of Onset and Evolution.--In half our cases the onset of severe symptoms was preceded by a prodromal period varying in length from two weeks to a few months, during which the patients complained of some headache, generally unilateral. The occurrence of a generalized seizure in Case 6 one year before the present illness cannot be taken as a prodromal sign of the present sub-cortical bleeding. In all the cases, as pointed out by Pilcher (1941), the actual onset of the illness was sudden : six times $(75 \%)$ with violent headache, associated mostly with vomiting, and followed immediately in five patients by impairment of consciousness or coma ; in one case the sudden onset consisted in a left motor hemiplegia developing within a few hours (Case 3) ; in another case too the onset was sudden and consisted in a grand mal seizure (Case 6). In only two patients was no initial impairment of consciousness observed-Cases 3 and 8 . David and Hécaen (1945) emphasize also the suddenness of onset and note as prominent features unilateral headache, vomiting, drowsiness, and at times epileptic discharges. Seizures were noted in the first phase only in two of our patients (Case 6 and

\footnotetext{
* The patient died suddenly 16 months after the operation. No necropsy could be obtained.
}

possibly Case 7). For Guillaume and Joinville (1945) the onset is not always so sudden; the intracerebral haematoma is said by these authors to develop in some cases slowly and to manifest itself by signs of intracranial hypertension with or without neurological disability.

To judge the evolution of the sub-cortical haematoma from surgically treated patients is hardly reliable. All we are able to say is that the very grave-looking onset with coma does not always lead to death ; this fact was pointed out many years ago by Penfield (1933). All our patients survived the onset of their illness, showing in the " second phase", as David and Hécaen (1945) put it, a great variety of neurological pictures. As far as we can tell from their pre-operative course, ranging in four cases from four to six weeks, the neurological disorders are apt to regress spontaneously to some extent during the first or second week, remaining stationary afterwards. One patient got worse after he had first regained consciousness and died in spite of the operation (Case 4).

Symptoms and Signs. - The most prominent symptom in our experience is the unilateral headache, which is excruciating at the onset; it corresponds to the side of the lesion and is probably due to acute distension of the dura by the expanding bleeding $\stackrel{\mathbb{Q}}{\square}$ within the brain. The neurological signs are웅 unilateral and generally localized to the side opposite $\varnothing$ to the headache. The focal signs depend on the site and size of the haemorrhage. Eye grounds were normal, except in Case 8 , in which blurring $\vec{f}$ of the optic discs developed in the second week. "Déviation conjugée" was observed in one patient (Case 5). Another important sign present in most cases is a stiffness of the neck. The C.S.F. has been found to be bloodstained in all cases in which the lumbar puncture was performed soon after the onset, and xanthochromic in the remaining cases. Blood pressure may be elevated on the first day owing to the acute increase of intracranial pressure (Case 8), but returns to a normal level within a week.

\section{Diagnosis}

Clinical Diagnosis.-From the foregoing considerations it appears that the main features characterizing spontaneous sub-cortical haematoma are the following: apoplectic onset; one-sided excruciating headache; contralateral neurological disability ; partial spontaneous recovery. That the unilateral headache (in our opinion a very important diagnostic feature) is not always present is shown in Case 3. In other words, we have first the picture of a sudden subarachnoid haemorrhage followed by the clinical signs of a unilateral expanding lesion. 
Ancillary Diagnostic Procedures.-The following help to confirm the diagnosis.

Lumbar puncture disclosed a subarachnoid haemorrhage in all our cases. However, cases occur which do not have blood in the C.S.F. (Russell, 1953).

Plain Radiographs of the Skull.--Signs of chronic intracranial hypertension are missing. Calcified lesions may occur (Jewesbury, 1947 ; Pilcher, 1941) or a shift of the pineal body (Pilcher, 1941).

Cerebral angiography is generally the next step because a ruptured aneurysm is often suspected, and may be carried out by the percutaneous method (Werner and Richter, 1950). The angiographic finding of an avascular intracerebral space-occupying lesion while there is evidence (from the proved subarachnoid haemorrhage) of a vascular lesion should definitely orientate the diagnosis towards that of an intracerebral haemorrhage. The arteriogram, as pointed out by Krayenbühl (1950) in our Case 1, can sometimes be misleading if a main cerebral artery fails to fill and there is very little displacement of the other vessels, leading to the diagnosis of thrombosis.

Pneumoencephalography may disclose a shift of the ventricular system with in some cases a well localized deformation of one lateral ventricle according to the location of the blood clot (Cases 7 and 8). But, of greater significance, the air study revealed in at least half the cases that the lesion was a huge intracerebral cavity communicating with the lateral ventricle. As shown in Case 1, the sub-cortical cavity can fail to fill with air on one occasion and fill well if pneumoencephalography be repeated after a week or two.

Electroencephalography discloses the hemisphere involved but fails generally to establish the exact localization. Repeated pre-operative electroencephalography, as in Case 7, shows that the noninvolved cerebral hemisphere develops an abnormal electrical activity after a few days, then recovers after a short time, while the diseased brain area shows depressed activity with better localization.

From the foregoing we see that the differential diagnosis of the spontaneous sub-cortical bleeding varies according to the stage at which the patient is seen. At the onset it must be differentiated from a ruptured aneurysm or from a capsular hemiplegia, occasionally from an arterial thrombosis, at the second stage from a brain tumour. Ancillary investigations are very useful but do not give any absolute answer to the question of the nature of the space-occupying lesion.
Aetiology and Pathogenesis

The question of aetiology has been dealt with at length by Jewesbury (1947), who emphasized the analogy between delayed traumatic intracerebral haematoma of Böllinger (1891), studied again by Symonds (1940), and spontaneous sub-cortical haemorrhage. No trauma has been recorded in our cases, though the last patient had made a strenuous effort three hours before his attack and during that time he had felt tired. But now that we know of such haematomas occurring without any traumatic background we must question the very existence of late post-traumatic haematoma. Systemic hypertension was absent and there was arteriosclerosis in only one case (Case 6), in which it certainly played a causative part as appears from the past history. In Case 8 the presence of a very abnormal occipital artery had raised the question of the intracranial arteries being abnormal too; the histological examination of some cortico-pial vessels from the excised cortex did not confirm this hypothesis. However, that arterial anomalies may account in some cases for apparently unexplained intracerebral bleeding has been illustrated by Kajtor's patient (1947-48) in whom the necropsy disclosed a hypoplasia of the anterior cerebral artery on the side of the bleeding. This author emphasizes that intracerebral bleeding may occur in the absence of thrombosis or embolism, being due to a localized vascular deficiency causing a local ischaemia of the brain. This idea had been already expressed by various authors, who pointed out that vasospasm is sufficient to cause a localized vascular deficiency (Westhphal and Baer, 1926; Hicks and Warren, 1951). The vasospasms would account for a prodromal period ; they would lead to a massive haemorrhage only if they recurred so frequently as to damage the arterial walls and the surrounding tissue. This theory accepts an arterial origin of the bleeding. Whether this is true in all cases is not yet proved. Craig and Adson (1936), struck by the fact that they were unable to find any active bleeding point after emptying the intracerebral cavity, sponsored the idea of a venous origin. We made the same observation in our unsuccessful search for an arterial bleeding source; however, we feel that slow venous oozing can hardly be responsible for the apoplectic onset observed. The subdural haematoma in Case 2 was due in our opinion to outpouring of blood from the intracerebral haemorrhage through the thin cortex. In certain instances, however, a venous origin due to increased intravenous pressure must be admitted, as in a recent case of post-traumatic arteriovenous fistula leading to a fatal intracerebral haemorrhage from distended veins. 


\section{Operative Treatment and Prognosis}

In all our cases an osteoplastic bone flap was turned, the cortex incised or excised, the cavity explored and emptied. There was one post-operative death, the seven other patients recovering from fairly to very well. The operative findings lead us to think that the simpler method of tapping the cavity and aspirating the fluid through a burr-hole (Huber and Schönbauer, 1951) or transdurally after lifting a small bone flap (David and Hécaen, 1945) are not satisfactory, as blood clots cannot be aspirated in these ways. Exact haemostasis should amply compensate for the risk of a secondary haemorrhage mentioned by the French authors. Nor are cases of spontaneous sub-cortical haematoma to be compared with acute post-traumatic intracerebral haemorrhage in which Kristiansen (1948) deems an osteoplastic craniotomy inadvisable as too traumatizing, advising instead simple enlargement of a burr-hole. Neither do we see the point of subjecting the patients first to a decompression and later, when the clots have eventually liquefied, to a second procedure (David and Hécaen, 1945). It seems to us that the foreign body which the sub-cortical haematoma represents has to be evacuated as soon as its presence has been ascertained.* A mere decompression, as advocated by Fazio (1950), in the apoplectic forms in which the patient's condition would not allow exact localization through ventriculography, is, in our opinion, of no more value than in cases of brain tumour.

The prognosis depends a good deal on the localization of the sub-cortical haematoma ; indeed, if such cases are recognized and adequately treated, there is not so much danger in the immediate future to life as there is to function.

\section{Pathology}

In contradistinction to Guillaume and Joinville (1945), who found that the majority of spontaneous intracerebral haematomas were located in the parieto-occipital region, we have a fairly equal proportion of anteriorly and posteriorly placed lesions.

Penfield's remark about the discoloration of the cortex overlying a sub-cortical haematoma was confirmed in at least half the cases. In three cases (Case 1, 6, and 7) the operator thought he had found neoplastic tissue, but each time the histological examination disproved it. It has been stated (Bagley, 1932) that formation of a cyst with organization of

\footnotetext{
* We thus fully agree with Diana Beck (1952), whose important contribution to the subject was unknown to the author at the time of writing this paper.
}

surrounding tissue after immediate outpouring of blood should begin within the first few days ; it is therefore of interest to note that a capsule around the sub-cortical haematoma was found only in two cases (Cases 1 and 3), being in the first six weeks and in the second five weeks old. The other intracerebral haemorrhages of our group did not show any membrane, at least to be recognized macroscopically at operation. David and Hécaen (1945) state that liquefaction of the blood clots should be almost complete after two weeks so as to justify surgical treatment by tapping of the cavity only. In three patients (Cases 1, 2, and 7) in whom the haemorrhage was older than four weeks the intracerebral cavity still contained blood clots, partially organized, and a certain amount of fluid. From our cases we cannot tell what will occur to the fluid-filled cavities. Rowbotham and Ogilvie (1945) think that osmotic pressure will increase as the coagula break down, so that fluid will get into the cyst from the surrounding tissue, as happens in chronic subdural haematoma. If this is really the case the complete resection of the cyst, membrane and contents, as outlined above, seems still more advisable; and conservative treatment should have very little prospect of success, since the fluid content will increase rather than decrease. As pointed out by Guillaume and Joinville (1945), among others, the haemorrhage does not generally destroy the cerebral parenchyma; this accounts certainly for the good recovery of function in some patients (Cases $1,2,3$, 6 , and 8), while destruction of neural pathways has certainly occurred in deeper regions and more centrally located haemorrhages, as in Case 5. In our second case, there was a thin subdural membrane adherent to the cortex overlying the intracerebral haematoma, a remnant of blood having leaked through the cortex from the subcortical haemorrhage.

\section{Summary}

Eight cases of spontaneous sub-cortical haematoma treated by craniotomy and evacuation of the cavity are presented. In seven instances no aetiological factor could be found; in one case arteriosclerosis may have played a part. The clinical picture consists mainly of the sudden onset of excruciating unilateral headache, followed by vomiting and impairment of consciousness, then spontaneous recovery from the initial stroke with unilateral neurological disability on the side opposite to the headache. Ancillary investigations are necessary : lumbar puncture to show fresh subarachnoid haemorrhage; arteriography to eliminate a ruptured aneurysm and to show an avascular 
intracerebral space-occupying lesion; pneumoencephalography to confirm the exact location of the lesion in disclosing in half the cases an air-filled cavity communicating with the lateral ventricle. In my opinion the sub-cortical haematoma has to be treated like any other sub-cortical space-occupying lesion, by complete removal.

Aetiology, pathogenesis, and prognosis are briefly discussed.

I wish to express my heartfelt thanks to Professor Hugo Krayenbühl, my former chief, for his kind permission to publish the cases of spontaneous subcortical haematoma which were observed in his clinic until the end of 1951.

\section{REFERENCES}

Bagley, C. (1932). Arch. Neurol. Psychiat., Chicago, 27, 1133. Beck, D. (1952). Communication at a meeting of the Society of British Neurological Surgeons, Zürich, June 6, 1952.
Böllinger, O. (1891). Internationale Beiträge zur wissenschaftlichen Medicin, Festschrift, Rudolf Virchow, vol 2, p. 459. Hirschwald,

Craig, W. McK., and Adson, A. W. (1936). Arch. Neurol. Psychiat., Chicago, 35, 701.

Cushing, H. (1903). Amer. J. med. Sci., 125, 1017, cited by Jewesbury.

David, M., and Hécaen, H. (1945). Presse méd. 53, 371.

Fazio, C. (1950). Sci. med. ital., 1, 101.

Guillaume, J., and Joinville R. (1945). Presse méd. 53, 606

Guicks, S. P., and Woinville, R. (1951). Arch. Path. Chicago, 52, 403.

Huber, K., and Schönbauer, L. (1951). Act. neurochir., Wien, 2, 81.

Huber, K., and Schönbauer, L. (1951). Act.
Jewesbury, E. C. O. (1947). Brain, 70, 274.

Kajtor, F. (1947-48). Confin. neurol., Basel, 8, 172.

Klemme, R. M. (1941). Cited by Jewesbury. Surgery, 10, 434.

Krayenbühl, H. (1950). Dtsch. med. Wschr., 75, 1177.

Kristiansen, K. (1948). Surgery, 24, 755.

Paula Nogueira, H. de (1949). Gaz. méd. port., 2, 990.

Penfield, W. (1933). Canad. med. Ass. J., 28, 369.

(1936). Arch. Neurol. Psychiat., Chicago, 35, 716.

Pilcher, C. (1941). Ibid., 46, 416.

Rowbotham, G. F., and Ogilvie, A. G. (1945). Brit. med. J., 1, 146

Russell, A. E., and Sargent, P. (1909). Proc. roy. Soc. Med., 2 , (Neurol Sect). 44, cited by Jewesbury.

Russell, W. R. (1953) Personal communication.

Symonds, C. P. (1940). Brit. med. J., 1, 1048, cited by Jewesbury.

Westhphal, K., and Baer, R. (1926). Disch. Arch. klin. Med., 151, 1.

Westhphal, K., and Baer, R. (1926). Dtsch. Arch. klin. Med., 151, 1. .
Werner, A., Babel, J., and Richard, P. (1952). Rev. méd. Suisse rom., 72,168

—, and Richter, H. (1950). Schweiz. med. Wschr., 80, 256. 